UCRL-TR-218854

\title{
Magnetism In 3d Transition Metals at High Pressures
}

V. Iota

February 2, 2006

LDRD final report 


\section{Disclaimer}

This document was prepared as an account of work sponsored by an agency of the United States Government. Neither the United States Government nor the University of California nor any of their employees, makes any warranty, express or implied, or assumes any legal liability or responsibility for the accuracy, completeness, or usefulness of any information, apparatus, product, or process disclosed, or represents that its use would not infringe privately owned rights. Reference herein to any specific commercial product, process, or service by trade name, trademark, manufacturer, or otherwise, does not necessarily constitute or imply its endorsement, recommendation, or favoring by the United States Government or the University of California. The views and opinions of authors expressed herein do not necessarily state or reflect those of the United States Government or the University of California, and shall not be used for advertising or product endorsement purposes.

\section{Auspices Statement}

This work was performed under the auspices of the U. S. Department of Energy (DOE) by the University of California, Lawrence Livermore National Laboratory (LLNL) under Contract No. W-7405-Eng-48. The project (02-ERD-046) was funded by the Laboratory Directed Research and Development Program at LLNL. 


\title{
FY05 LDRD Final Report Magnetic Transition Metals and Oxides at High Pressures \\ LDRD Project Tracking Code: 02-ERD-046 Valentin I ota, Principal Investigator
}

\begin{abstract}
This research project examined the changes in electronic and magnetic properties of transition metals and oxides under applied pressures, focusing on complex relationship between magnetism and phase stability in these correlated electron systems.

As part of this LDRD project, we developed new measurement techniques and adapted synchrotron-based electronic and magnetic measurements for use in the diamond anvil cell. We have performed state-of-the-art X-ray spectroscopy experiments at the dedicated high-pressure beamline HP-CAT (Sector 16 Advanced Photon Source, Argonne National Laboratory), maintained in collaboration with of University of Nevada, Las Vegas and Geophysical Laboratory of The Carnegie Institution of Washington.

Using these advanced measurements, we determined the evolution of the magnetic order in the ferromagnetic $3 \mathrm{~d}$ transition metals (Fe, Co and $\mathrm{Ni}$ ) under pressure, and found that at high densities, $3 \mathrm{~d}$ band broadening results in diminished long range magnetic coupling. Our experiments have allowed us to paint a unified picture of the effects of pressure on the evolution of magnetic spin in $3 \mathrm{~d}$ electron systems.

The technical and scientific advances made during this LDRD project have been reported at a number of scientific meetings and conferences, and have been submitted for publication in technical journals.

Both the technical advances and the physical understanding of correlated systems derived from this LDRD are being applied to research on the $4 \mathrm{f}$ and $5 \mathrm{f}$ electron systems under pressure.
\end{abstract}




\section{Introduction/ Background}

The electronic and magnetic interactions in transition metals are greatly affected by the spatial arrangement of the atoms in their solid phases. The strong inter-correlation between electronic structure and atomic configuration in 3-d metals is of great fundamental importance in condensed matter physics. In the case of the $3 \mathrm{~d}$ magnetic metals ( $\mathrm{Fe}, \mathrm{Co}, \mathrm{Ni})$ and their oxides, it is believed that the polarization of the valence band holds the key to understanding the stability and the crystal structure of the element at ambient conditions [1].

By modifying the interatomic distances and potentials, applied hydrostatic pressure affects the band structure and magnetic interactions in solids. Therefore, high pressure measurements of electronic band structure combined with measurements of crystal structure can be used to quantify the contribution of the magnetic polarization to phase stability in narrow d-band metals and to understand the nature of magnetic ordering in ferromagnetic solids. In addition, understanding the contributions of the magnetic interactions to phase stability at high pressure is essential for predicting the stability and properties of planetary interior components at Earth core conditions [2, 3].

Moreover, the study of pressure-induced phase transitions in 3-d metals and oxides may offer physical insight into technologically important properties of magnetic materials. By inducing changes in inter-atomic bond lengths, applied pressure can force transition metals into metastable configurations, such as ones reported in Co and Fe nanophases. Understanding the kinetics of phase transitions from stable to metastable structures and from magnetic to nonmagnetic phases, provides understanding of the fundamental variables controlling these changes, and offers the possibility of using those variables for engineering new materials.

\section{Research Activities}

In order to measure the magnetic moments of sample under hydrostatic pressures, we perfected a combination of X-ray spectroscopy measurements that allowed us to determine the electronic configuration, and the magnetic moments of magnetic $3 \mathrm{~d}$ metals and oxides under pressure.

Among the advanced measurements we carried out are high-resolution X-ray Emission Spectroscopy (XES), and X-ray Magnetic Circular Dichroism (XMCD). As detailed below, we were able to solve the issue of signal absorption by the anvils in X-ray spectroscopy by using bright focused $3^{\text {rd }}$ generation synchrotron sources and by measuring the $\mathrm{K}_{\beta}$ emission in a radial geometry through beryllium gaskets.

One of the difficulties in extending $\mathrm{X}$-ray spectroscopic techniques to the DAC has been the strong absorption of the diamond anvils below $10 \mathrm{keV}$. To avoid the attenuation

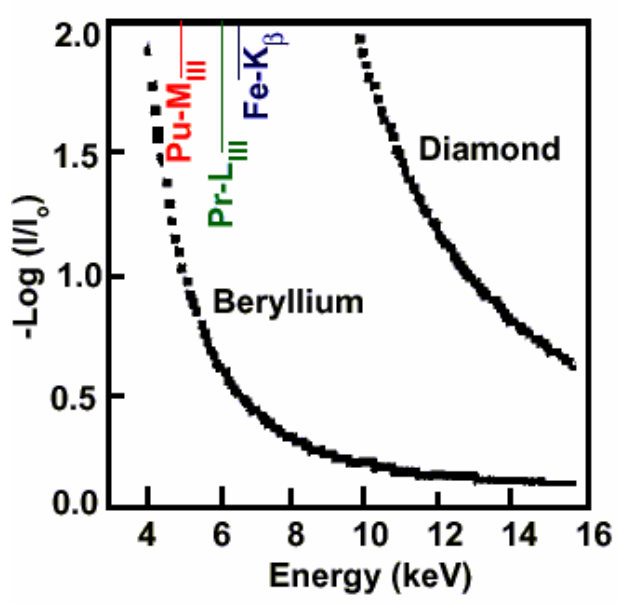

Fig 1: The use of Be gasket extends the range of XES-DAC measurements to include the 4$10 \mathrm{keV}$ range (covering the $\mathrm{K}$ emissions from $3 d$ transition metals, the $\mathrm{L}$ lines in $4 f$ rare earths as well as the $\mathrm{M}$ lines in $5 f$ actinides). 
of the signal in the anvils, we were able to use X-ray transparent gaskets made from high strength beryllium metal, allowing us to measure the K emission energy in radial geometry. This technique extends the range of measurable energies to cover energies down to $3 \mathrm{keV}$, and will permit electronic measurements on $3 \mathrm{~d}$, $4 \mathrm{f}$ and $5 \mathrm{f}$ elements.

In addition, in collaboration with scientists at the APS Polarization Studies Group (beamline 4ID-D) we have developed XMCD measurements in a diamond anvil cell, by using high resolution monochromatic focused beams in conjunction with a specially designed diamond anvil cell - using partially perforated diamonds. A brief description of these two measurements is presented below.

\section{X-ray Emission Spectroscopy (XES) in the diamond anvil cell:}

In X-ray Emission Spectroscopy (XES) $1 s$ core-electrons are excited by highenergy "white" $\mathrm{x}$-rays from an intense synchrotron source. The x-ray emissions are

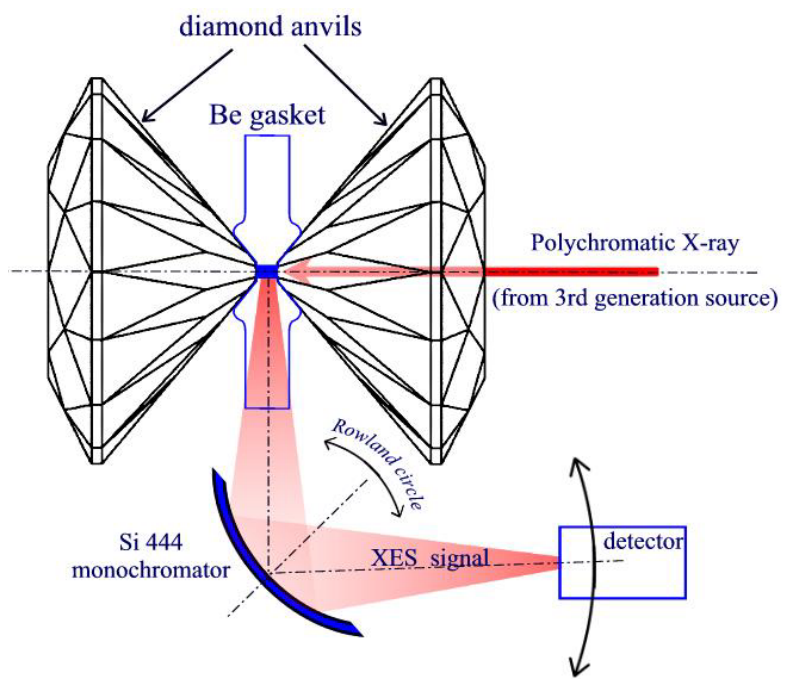

Fig 2: Experimental set-up for DAC-XES using pre-formed beryllium gaskets in axial geometry.

as $\Delta \mathrm{E}=\mathrm{J}(2 \mathrm{~S}+1)$, and $\mathrm{I}^{\prime} / \mathrm{I}=2 \mathrm{~S} /(2 \mathrm{~S}+2)[4]$. analyzed using a high-resolution (1eV) crystal analyzer to determine the spin electronic state of the sample. Because the final state of the fluorescence process is a one-hole state, XES directly probes the electronic density of states (DOS) of the valence band and is sensitive to the magnetic polarization of the $3 \mathrm{~d}$ band as well as to chemical shifts in the core-level binding energies of the sample. For the 3-d transition elements, the $K_{\beta}$ line $(3 p \rightarrow 1 s$ transition) contains information about the polarization of the valence band via the $3 p$ $3 d$ exchange interaction. The separation, $\Delta \mathrm{E}$, and intensity ratio, I'/I, of these multiplet transitions, can be used to determine the number of unpaired electrons in d-states, $2 \mathrm{~S}$, and the exchange integral, J,

\section{X-Ray Magnetic Circular Dichroism (XMCD) in the Diamond Anvil Cell}

X-ray Magnetic Circular Dichroism (XMCD) is an element-sensitive probe of the localized magnetic moments by using polarized synchrotron radiation and quarter-wave plates to measure the spin-dependent $\mathrm{K}_{\beta}$ emission fine structures. However, while XES is sensitive to the total magnetization of the measured system, XMCD can discern between the contributions of different atoms in an alloy or multilayer, or between their orbital and spin moments.

The XMCD process is illustrated in the diagram in figure 3 below. For magnetic materials in the presence of an applied magnetic field, the spin up and spin down bands 
are not equally populated. For an applied field in the "up" direction, there will be some empty spin up $3 \mathrm{~d}$ states. In this case, because of conservation of spin, only the electrons with up spin can be excited into the $3 d$ states. When the orbital motion of the $2 p$ states is in the same sense as the circular motion of the incident light the transition probability is larger; when the two motions are in opposite directions the transition probability is smaller. The XMCD is the difference, for a magnetic material, between the absorption of left and right circularly polarized $X$-rays.

In XMCD, the atom absorbs a photon, giving rise to the transition of a core electron to an empty state above the Fermi level. The absorption cross-sections are large, especially in the energy range characteristic of $3 \mathrm{~d}$ elements $(6-8 \mathrm{keV})$. The absorption edges have energies which are characteristic for each element and, due to the dipole selection rules, final states with different symmetries can be probed by choosing the initial state.

As with XES, the limiting factor in the traditional XMCD experiments in $3 \mathrm{~d}$ elements is that the $\mathrm{K}$ absorption edge $(\sim 7 \mathrm{keV})$ is strongly attenuated by the diamond anvils. In addition, the small band splitting require the use of high-intensity x-rays and highresolution detectors, unavailable until recently for use in the DAC. To avoid the absorption of XMCD signal by the diamond-anvil, the spectroscopy experiments will be conducted in a radial configuration, using hardened $\mathrm{Be}$ gaskets. This allows simultaneous access along the axial direction, for use for in-situ laserheating, on-line ruby pressure measurements, or concurrent $\mathrm{X}$-ray diffraction measurements. In addition, XMCD measurements require the use of external polarizing magnetic fields: to avoid interference from the DAC, the high pressure XMCD experiments will be conducted using non-magnetic (Be-Cu alloy) cells. 


\section{Results/ Technical Outcome}

\section{1) Magnetic transitions in iron at high pressure}

In iron, we have measured the $\mathrm{K}_{\beta}$ emission across the $\alpha(b c c) \rightarrow \varepsilon(h c p)$ structural transition occurring at $13 \mathrm{GPa}$ at room temperature. In the low pressure phase ( $\alpha$-ferromagnetic), in addition to the main, narrow $\mathrm{K}_{\beta}{ }^{1-3}$ peak, we observe a low energy satellite $\left(\mathrm{K}_{\beta}{ }^{\prime}\right)$ - associated with transitions into the down spin state of the $3 \mathrm{p}$ orbital (i.e. in the final-state of the transition the total spin of the $3 \mathrm{p}$ hole is aligned opposite to the magnetic moment of the valence band).

Figure 4 shows the disappearance of the $\mathrm{K}_{\beta}$, satellite in the high pressure phase of iron (blue spectrum in figure 4). This indicates the quenching of the magnetic moment of the Fe $3 \mathrm{~d}$ valence electrons in the high pressure phase suggesting that the $\alpha \rightarrow \varepsilon$ structural transition is associated with an electronic transition from a high spin state to a low-spin state. $\varepsilon$-Fe is nonmagnetic. This result confirms the recent report by Rueff et al. [4] that the high pressure phase is nonmagnetic and contradicts assertions (based on direct magnetization measurements) that $\varepsilon-F e$ may be ferromagnetic [5].

By combining spectroscopy measurements with X-ray diffraction measurements we were able to determine in detail the electronic, magnetic and structural phase boundaries for the $\alpha-\varepsilon$ phase transition in iron $(\mathrm{Fe})$. While our XMCD data show that the long range magnetic order (the ferromagnetism) is suppressed promptly upon the structural transition, our XES measurements reveal a diminished but measurable remnant spin in hep-Fe up to $35 \mathrm{GPa}$. Our result is inconsistent with the proposed model of an electronically driven $\alpha-\varepsilon$ transition, and suggests the possibility of an antiferromagnetic or paramagnetic state of hexagonal iron at high pressures.

This result may have important consequences for current geophysical models of Earth's magnetic field, since hexagonal iron is believed to be one of the major constituents of our planet's inner core $[2,5]$.

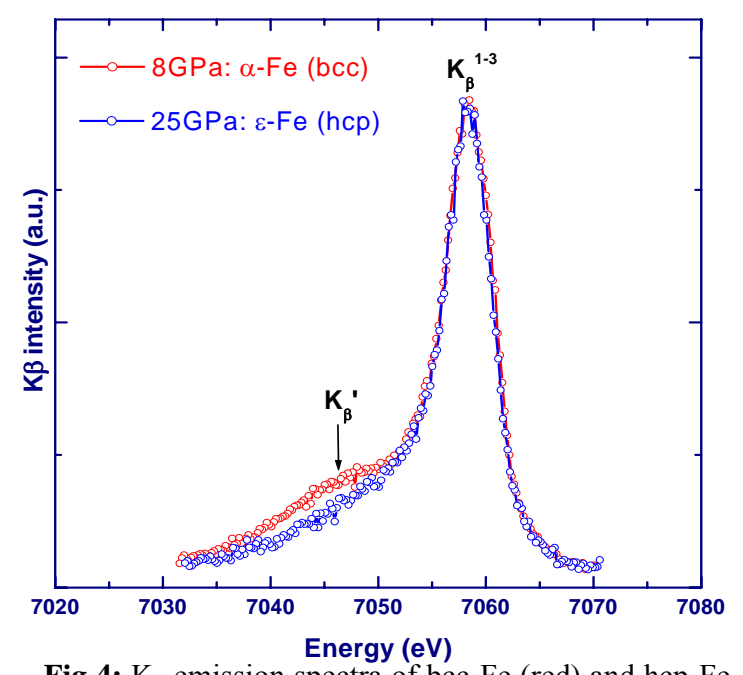

Fig 4: $\mathrm{K}_{\beta}$ emission spectra of bcc-Fe (red) and hcp-Fe (blue); the disappearance of the low energy satellite $\mathrm{K}_{\beta}$ ' across the phase boundary suggests the high pressure phase is non-magnetic.

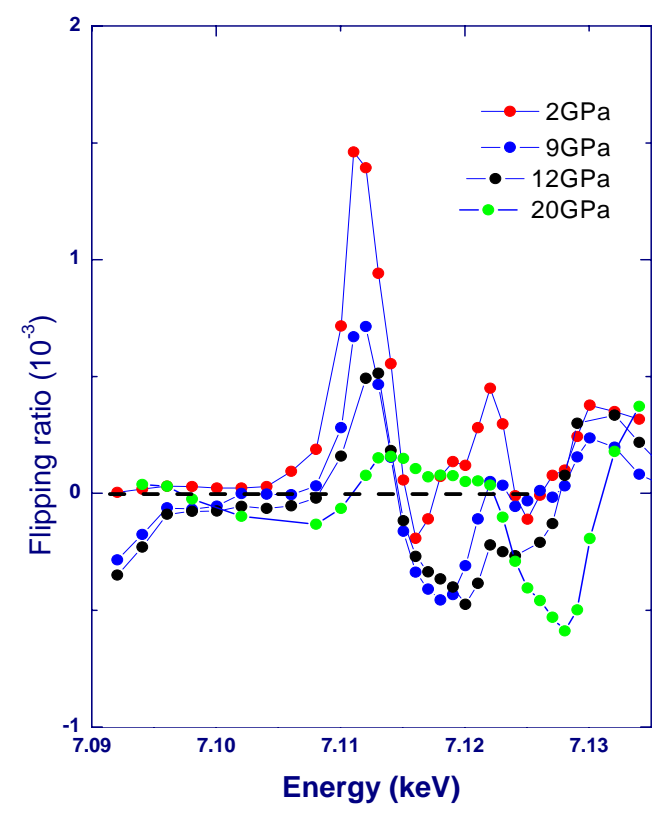

Fig 5: XMCD measurements in compressed iron show the sharp loss of long range magnetic order across the bcc-hcp structural transition. 


\section{2) Correlation-driven phase transitions dense cobalt}

We investigated the spin state of cobalt up to a pressure of $125 \mathrm{GPa}$ by monitoring the $\mathrm{K}_{\beta}$ emission of Co $(7648 \mathrm{eV})$. As in the case of $\mathrm{Fe}$, the low energy shoulder of the emission band results (labeled $\mathrm{K}_{\beta}$ ' in figure 5) results from the exchange interaction between the (3p $\uparrow)$ final hole state and the spin polarized $3 \mathrm{~d}$ band. For pressures below $\sim 90 \mathrm{GPa}$, the $\mathrm{K}_{\beta}$ line shape remains unchanged; however, above $95 \mathrm{GPa}$, and up to the maximum pressure of the experiment $(125 \mathrm{GPa})$ we observed a clear reduction in the intensity of the $\mathrm{K}_{\beta}$ ' emission. This result suggests the gradual disappearance of the $3 \mathrm{~d}$ net spin in Co above 95GPa.

$\mathrm{X}$-ray spectroscopy in high-pressure cobalt shows the quenching of both the ferromagnetism and the electronic spin at the $\varepsilon-\beta$ phase boundary. However, in contrast

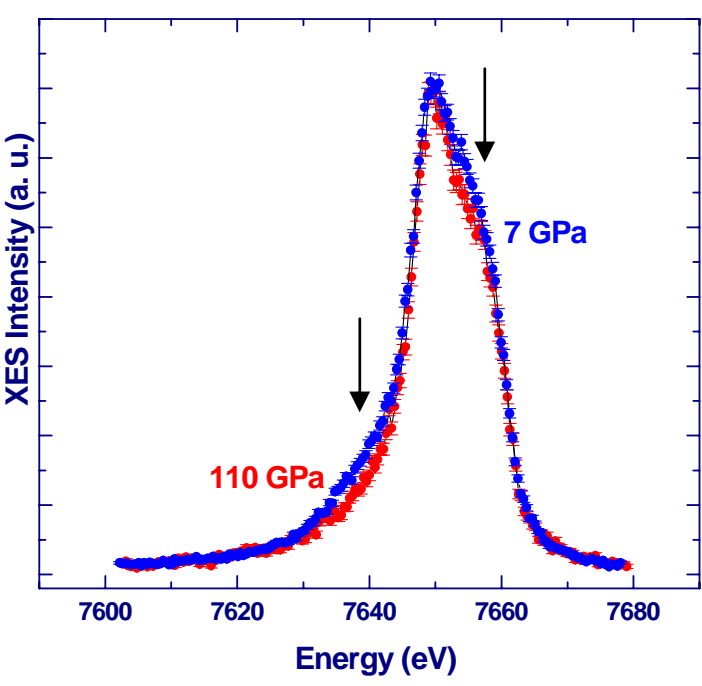

Fig 6: $K_{\beta}$ emission of cobalt: spectra are collected before (red) and after (blue) laser heating to $1800 \mathrm{~K}$. The differences suggest electronic configuration changes accompany the $\varepsilon-\gamma$ structural phase change. with our result in iron, the structural transition is preceded by a significant suppression of the $3 \mathrm{~d}$ spin. This result suggests an electronically-driven transition, where the pressureinduced increase in the $3 \mathrm{~d}$ bandwidth is an important factor in the high pressure transformation to fcc-Co.

By simultaneously measuring angle resolved X-ray diffraction patterns of the sample, were able to correlate these changes in electronic structure with the $\varepsilon(\mathrm{hcp})$ to $\beta(\mathrm{fcc})$ structural phase transition in cobalt which occurs over an extended pressure range between 100-150GPa [6,7]. A comparison of the sequence of XES and XRD spectra, shows that the changes in electronic configuration precede the $\varepsilon-\beta$ phase transition. This suggests that the hcpfcc structural transformation in cobalt is triggered by pressure induced changes in the electronic configuration. A mechanism to account for the initiation of the structural transformation proposes that applied pressure induces a broadening of the $3 \mathrm{~d}$ valence band, diminishing the electron correlations and reducing the site to site repulsions.

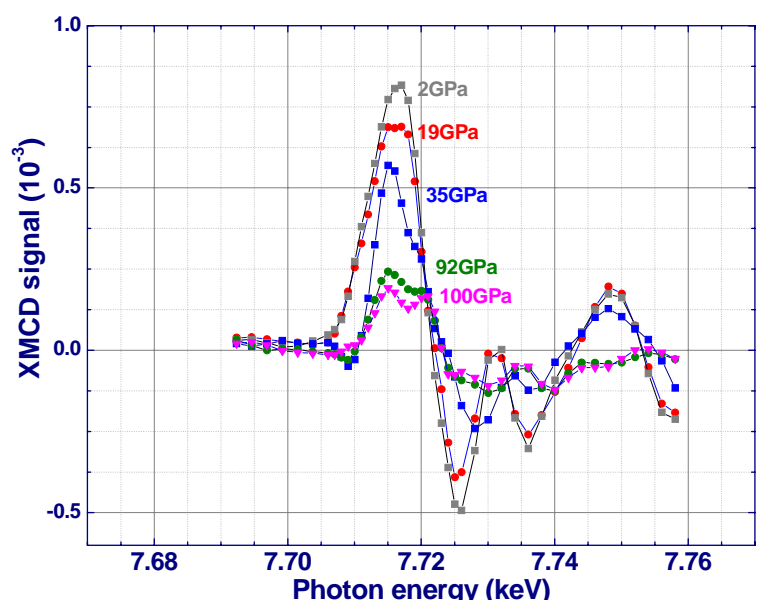

Fig 7: XMCD in compressed cobalt. The diminished dichroism with pressure suggests a reduced long range magnetic order. However, hcp-Co remains ferromagnetic up to $100 \mathrm{Gpa}$. The differences suggest electronic configuration changes accompany the $\varepsilon-\gamma$ structural phase change 
3) Evidence for a nonmagnetic nickel at high pressures

We performed XES measurements on nickel at pressures up to $70 \mathrm{GPa}$, trying to determine the existence (and the boundaries) of a non-magnetic high density phase of $\mathrm{Ni}$. The results are presented We performed $\mathrm{K} \beta$ emission measurements on compressed nickel at pressures up to $70 \mathrm{GPa}$, attempting to determine the existence (and the boundaries) of a non-magnetic high density phase of $\mathrm{Ni}$. The results are presented in Fig 8.

One of the pitfalls of using XES to ascertain the magnetic (spin) state of $\mathrm{Ni}$ is that there is no obvious splitting in the $\mathrm{Ni} \mathrm{K}_{\beta}$ band. The main reason for this is that the $3 \mathrm{~d}$ valence band of $\mathrm{Ni}$ is almost full ( 8 spins). As a result, the net spin is much lower in Ni than either in $\mathrm{Fe}\left(\sim 3 \mu_{\mathrm{b}}\right)$ or in Co $\left(2.5 \mu_{\mathrm{b}}\right)$. This is reflected in weaker exchange interaction between the $3 p$ hole and the valence band spin, resulting is a more symmetric lineshape for the $\mathrm{Ni} \mathrm{K}_{\beta}$. In conclusion, the X-ray Emission Spectroscopy of the $\mathrm{K} \beta$ line is not sensitive enough to changes in spin state; to the limit of our sensitivity observe no changes in the line shape up to the maximum pressure (70GPa).

However, our subsequent XMCD measurements in nickel up to $70 \mathrm{GPa}$ show a monotonous decrease in the amplitude of the magnetic moment with pressure, suggesting the existence of a nonmagnetic nickel state above $150 \mathrm{GPa}$.

Importantly, our results clearly show an increased $3 d-4 p$ hybridization with pressure in all three metals studied, suggesting that the main mechanism for magnetism suppression is band broadening of the $3 \mathrm{~d}$ valence band [8].

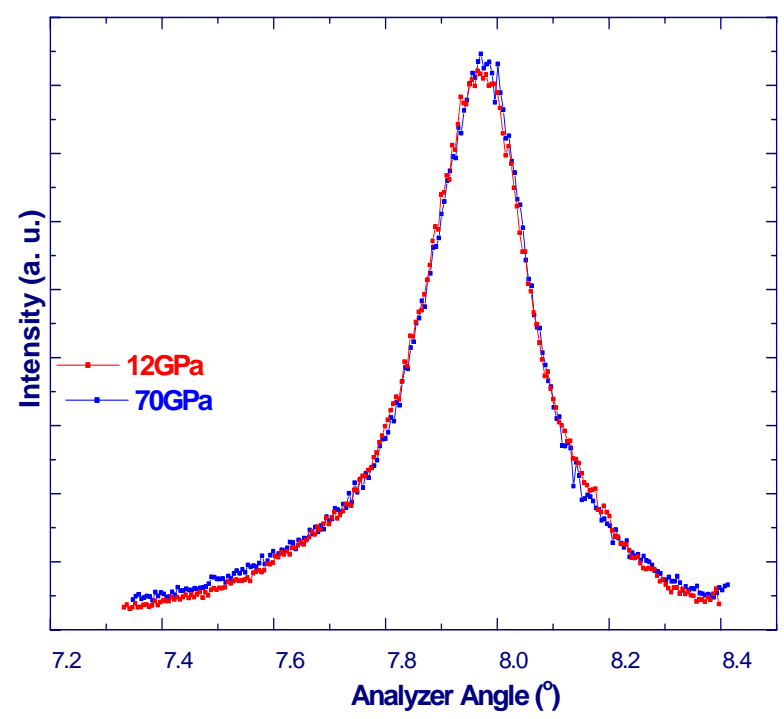

Fig 8: $\mathrm{K}_{\beta}$ emission of nickel up to $70 \mathrm{GPa}$. No changes are observed over the entire pressure range of the experiment. However, since Ni has a low net spin, the spitting of the $\mathrm{K}_{\beta}$ emission may to weak to determine the polarization of the d-band.

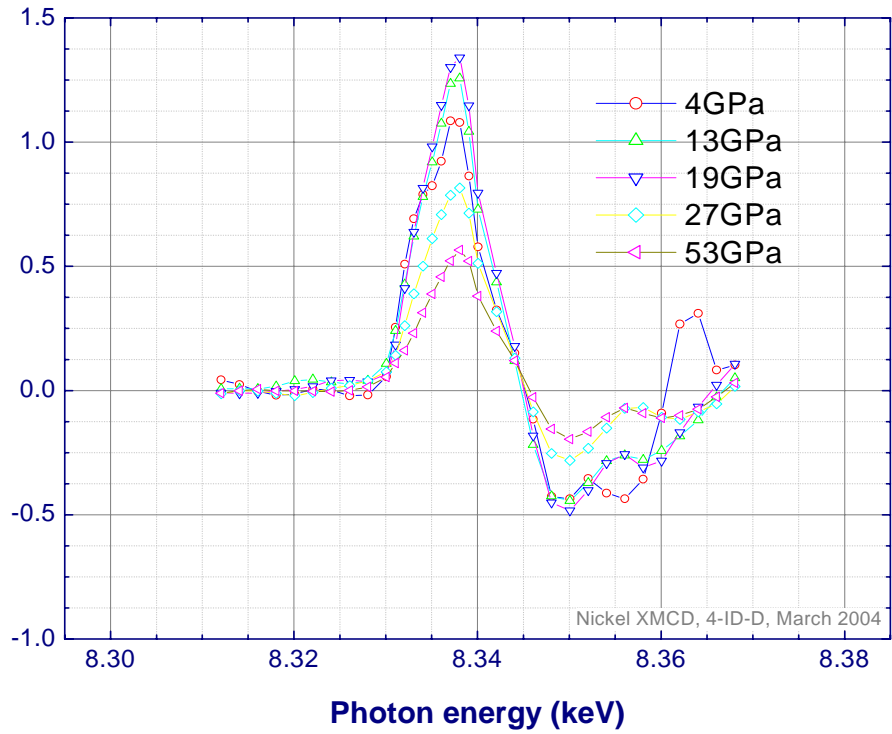

Fig 9: $\mathrm{XMCD}$ of nickel up to $70 \mathrm{GPa}$. We observe a diminished XMCD signal suggesting decreased ferromagnetism in compressed $\mathrm{Ni}-$ due presumably to pressure induced band broadening. 


\section{Exit Plan}

The measurement techniques and the scientific advances made during this LDRD project can be utilized to solve other important physics problems. Currently, similar Xray spectroscopy experiments are being used and further developed to address the complex physics issues surrounding volume collapse transitions in rare earth metals [9]. In the future, this study offers a template for studying other correlated electron systems under pressure, such as program related materials.

\section{Summary}

In this study we have investigate the electronic and magnetic properties of $3 \mathrm{~d}-$ metals and their oxides at high pressures. The primary focus has been the study of spin transitions in iron $(\mathrm{Fe})$ cobalt $(\mathrm{Co})$ and nickel $(\mathrm{Ni})$ and to understand he correlations between magnetism and structural properties in these $3 \mathrm{~d}$ transition metals.

Our results show diminished magnetic spin and ferromagnetic order with pressure in all three magnetic metals studied. Based on our measurements we propose that the principal mechanism for magnetism suppression in the pressure-induced broadening of the $3 \mathrm{~d}$ valence band.

\section{References}

[1] P. Soderlind et al., Phys. Rev. B 50, 5918 (1994); Phys. Rev. B 53, 14063 (1996).

[2] H.K. Mao, et al. J. Appl. Phys. 38, 272 (1967); Song, Nature (1997)

[3] H. Hasegawa and D. G. Pettifor, Phys. Rev. Lett. 50, 130 (1983).

[4] J. P. Rueff, et al. Phys Rev B. 60, 4510 (1999); G. Fiquet et al. Science 291, 468 (2001).

[5] S tuart Gilder and Jonathan Glen, Science 279, 72 (1998)

[6] C.S. Yoo, H. Cynn, P. Soderlind, V. Iota, Phys. Rev. Lett. 84, 4132 (2000)

[7] C. S. Yoo, P. Soderlind and H. Cynn, J. Phys. Condens. Matter 10, L311 (1998)

[8] V. Iota et al, (under review)

[9] A. K. McMahan et al, J. Comp. Aid. Mat. 5, 131 (1998). 